\title{
The limit to behavioral inertia and the power of default in voluntary contribution games
}

\author{
Jia Liu' ${ }^{1}$ - Yohanes E. Riyanto ${ }^{2}$
}

Received: 2 November 2015 / Accepted: 14 February 2017 / Published online: 23 February 2017 (C) The Author(s) 2017. This article is published with open access at Springerlink.com

\begin{abstract}
It is well documented that people are reluctant to switch from a default option. We experimentally test the robustness of this behavioral inertia in a collective decision-making setting by varying the default option type and the decision-making environment. We examine the impacts of automatic-participation and no-participation default options on subjects' participation in a public goods provision and their contributions. Two variants of public goods game are employed: the linear and the threshold public goods games. The study shows the evidence of partial stickiness rather than complete stickiness of default options as indicated in empirical studies. Our experimental results square with the evidence of behavioral inertia only when the automatic-participation default is used. This default boosts contributions in the linear public goods game but not in the threshold public goods game. The evidence
\end{abstract}

We thank Tim Cason, David Gill, Dan Houser, Juanjuan Meng, Andreas Ortmann, the editors, two anonymous referees and seminar participants at NTU, the International Meeting of the Economic Science Association (ESA) at New York University, the Australian Conference of Economists (ACE) in Melbourne, the Econometric Society North American Meeting at the University of Southern California, and the Asian Meeting of the Econometric Society at the National University of Singapore for their useful comments.

Electronic supplementary material The online version of this article (doi:10.1007/s00355-017-1036-x) contains supplementary material, which is available to authorized users.

Jia Liu

jia.liu@newcastle.ac.uk

Yohanes E. Riyanto

yeriyanto@ntu.edu.sg

1 Newcastle University Business School, 5 Barrack Road, Newcastle upon Tyne NE1 4SE, UK

2 Division of Economics, Nanyang Technological University, 14 Nanyang Drive HSS-04-70, Singapore 637332, Singapore 
of partial stickiness is robust to the variation of the game employed, but the effect on contribution is sensitive to it.

\section{Introduction}

There is ample empirical evidence in the literature that people are reluctant to switch from a default option. Madrian and Shea (2001), Thaler and Sunstein (2003) and Choi et al. (2004) show that the use of automatic enrollment instead of non-enrollment as the default choice in the $401(\mathrm{k})$ saving for retirement program increases the enrollment rate because people are inclined to stay with the default option provided. Johnson et al. (1993) show that people are reluctant to switch from the stated default option when choosing insurance policies. In a similar vein, Johnson and Goldstein (2003) show that the use of opt-out schemes in organ donation drives, whereby automatic enrollment is set as the default, boosts the enrollment rate.

Several explanations are proposed in the literature to rationalize this behavioral inertia. For instance, the prevailing default option might be perceived as a suggestion of the course of action to follow (see Madrian and Shea 2001; Thaler and Sunstein 2008; Beshears et al. 2009). Switching from the default option might be costly. As a result, people prefer to stick with the default (see Samuelson and Zeckhauser 1988). People might be loss averse and reluctant to switch away from the status quo because switching causes a sense of loss relative to the initial reference point (see Kahneman et al. 1991). The existing explanations for people's inclination to stay with the default seem to suggest that the type of default option employed is inconsequential. That is, the tendency for people to exhibit affinity to the status quo choice is a general phenomenon and would likely persist regardless of the type of the default option employed.

In this paper, we delve into this issue by carefully investigating whether or not the type of default option employed is indeed irrelevant for people's decision to whether or not to stay with the default option through a series of controlled laboratory experiments. To achieve this goal, we vary the type of default option given to the subjects in our experiments. We focus our analysis on public goods experiments and on the default participation option in public goods provision. Specifically, our research objectives are threefold. Firstly, we evaluate the robustness of the evidence showing that people tend to stick to the default option. Secondly, we investigate the impact of different types of default option on people's incentive to cooperate in a collective setting. Thirdly, we evaluate whether the types of the public goods game employed influence the ability of the default participation option to affect the level of cooperation.

In particular, we focus on two variants of public goods game, namely the linear public goods game, where the unique Nash equilibrium is characterized by zero contribution, and the threshold public goods game, where a zero-contribution equilibrium and multiple interior Nash equilibria exist. Public goods provision with a threshold has desirable features in some circumstances and the presence of a provision point could lessen the free-riding problem (Bagnoli and Lipman 1989; Messer et al. 2008). The presence of a contribution threshold essentially transforms the prisoner's dilemma type situation that exists in the linear public goods game into a coordination problem. The consideration of the threshold public goods game would also allow us to further check 
the robustness of the results obtained under the linear public goods game. We conducted three between-subject experimental treatments for each variant of the public goods game, making six treatments altogether. The first treatment involves no default option, and serves as our control treatment. The second treatment is the opt-in treatment, in which by default people do not contribute to the public goods provision, but can decide to opt-in if they wish to do so. The third is the opt-out treatment, where by default people must make a non-zero contribution, but can decide to opt-out if they wish.

To the best of our knowledge, there are only a handful of recent papers related to ours. Messer et al. (2007) found that the use of a status quo of giving increased contribution in public goods games initially but the effect was not long lasting. Messer et al. (2008) framed their public goods contribution game as a collective contribution effort to provide generic advertising. In their framework, firms operating in agricultural sector can voluntarily participate in the provision of generic advertising which would bring collective benefits. They investigated the standard voluntary contribution mechanism and the provision point mechanism similar to ours. Messer et al. (2008) also labelled the parameters of the game using specific terms such as production quantity, production cost, industry price, etc. to make the game consistent with the collective generic advertising and promotion framing they used. They did not, however, analyzed the role of default (participation) options. Their focus was more on finding which mechanism out of these two worked better best in increasing contribution.

In contrast, we did not provide any specific contextual framing for the voluntary contribution mechanism. Rather, we presented it as a standard public goods contribution game. We provided information on subjects' benefit from the public goods contribution (i.e. the Marginal Per Capita Return/ MPCR) in both games, the refund policy and the rebate policy used in the threshold public goods game. Both Messer et al. (2008) and our paper produced the classic under-provision problem in voluntary contribution settings.

Altmann and Falk (2009) focused on the interplay between the use of the nonbinding default option, cognitive skills and the incentive to cooperate in the provision of public goods. They showed that the use of the non-binding option only influenced the behavior of people with low cognitive skills.

Carlsson et al. (2015) presented a field experiment of the public goods game in Vietnam. Their subjects were villagers in a rural village who had to make a collective monetary contribution to build a bridge, a vital transportation infrastructure for the villagers. They compared the use of the no default option with zero-contribution and full contribution default options. They showed that, relative to the full contribution default option, the zero-contribution default option decreased the average contribution by around $20 \%$.

Thus, the settings employed in both Messer et al. (2008) and Carlsson et al. (2015) were adapted to fit their specific scenarios. In contrast, our present paper adopts a standard public goods contribution setting. We opt for this setting because the voluntary contribution setting in its simplest form serves our purpose. We try to avoid any interaction effect between a specific game setting and the different types of defaults as the latter is the focus of this paper.

Finally, Cappelletti et al. (2014) explored channels through which a suggested contribution in a linear public goods game might influence the contribution decision. In particular, they varied the format of the suggested contribution (i.e. either it was presented as a default contribution or as a contribution advice with no default setting 
given) and the source of the suggested contribution level (i.e. either it was set by a human participant or set by a computer). They found that subjects were more likely to accept the suggested contribution level when it was presented as a default contribution rather than as a contribution advice. The focus of the analysis in Cappelletti et al. (2014) was twofold. Firstly, it was on the comparison of the role of default contribution amount vis-a-vis the role of contribution advice without default setting in motivating subjects to contribute to public goods provision. Secondly, it was on the evaluation of whether the source of the default contribution amount would encourage subjects to contribute more by comparing a default option set by a human participant and a default option set by a computer. Our paper, in contrast, focuses on the comparison of the role of different default participation options, rather than different default contribution amounts, in motivating subjects to make public goods contributions and also on the inertia of different default options implemented.

It should be noted that the default option used in the opt-in treatment in all of the papers mentioned above is similar to our opt-in treatment, but not with our opt-out treatment. In our opt-out treatment, if subjects do not opt out of the public goods contribution, they still have to consciously choose their non-zero contribution. In addition, each of these studies focused on one type of public goods game and they found differentiated effects of defaults, while our study focuses on both the linear and threshold public goods games, which allows us to test whether the effects are sensitive to the game type.

In the opt-out condition, subjects need to actively decide on the contribution amount even if they stay with the default. It is essentially a hybrid system of the conventional default option and a system of active decisions. In a seminal paper on saving behavior, Madrian and Shea (2001) found that defaults were powerful. On the same saving behavior, Carroll et al. (2009) instead found that requiring people to choose their amount of saving rather than forcing them to save a certain amount may have some positive effects on the overall saving rate. It was shown theoretically in Carroll et al. (2009) that the optimality of a default setting vis-à-vis an active decision setting depended on the homogeneity of the preferences. They indicated that, under some conditions, a hybrid system consisting of a default option and an active decision setting might be optimal, which could be a promising area for future research. Following the spirit of Carroll et al. (2009), we thus employ a hybrid-like system where both a default option and an active decision setting are present. In this context, our paper can be seen as an attempt towards this research direction.

Another reason is that we would like to still maintain the voluntary nature of contribution in the opt-out treatment when subjects decide to stay with the default setting rather than arbitrarily imposing a certain level of contribution on them. Likewise, we do not have any positive default contribution levels in the opt-in treatments. In the opt-in condition, subjects must first decide whether or not they want to participate. If they decide to participate in the voluntary contribution, they need to move away from the default condition and actively choose a positive contribution level. Otherwise they can remain in the default.

Our paper contributes to the literature in several ways. Firstly, to the best of our knowledge, this is the first study showing the evidence of partial stickiness, rather than complete stickiness, of default options. By manipulating the decision environment (i.e. 
the absence or presence of default option) and the type of defaults (i.e. participation or no-participation default), we are able to show that under some condition subjects would not always want to stay with the default option provided. Secondly, this paper employs the participation and no-participation default options, rather than the contribution amount default options, in the provision of public goods. Thus, the default option provided in this paper still preserves the voluntary nature of public goods provision. That is, even when the default option employed is participation, subjects would still have to decide the (positive) amount of contribution. Thirdly, this paper also presents the sensitivity analysis of the impact of default participation option on the public goods contribution to the types of voluntary contribution games employed (i.e., the linear public goods game and the threshold public goods game).

We show that the nature of a default option influences subjects' decision on whether or not to stay with it. We also find that the level of contribution under different default options is sensitive to the type of the public goods game employed. Contrary to the existing evidence, subjects in our experiments do not always stick to the default options. They consciously make an active decision to nullify the default option under the optin system, but not under the opt-out system. Essentially, under the opt-in system, the default option induces people to free-ride on others' contributions, and people are averse to being perceived as free-riders. As a result, they would prefer to switch from the default decision. This behavior is robust to the type of the public goods game employed.

The use of the opt-out system also results in significantly higher individual contributions and individuals' earnings beyond that under the opt-in and the baseline settings. However, this only happens in the linear public goods game. It has the opposite effects in the threshold public goods game. A possible explanation for this result is that the presence of the threshold makes it more or less a coordination problem. The presence of the default requiring a decision on the contribution level might add an additional layer of complications to the context and might make people focus more on meeting the threshold instead of achieving the socially optimal outcome. In other words, the effectiveness of the cooperative default on the contribution level is sensitive to the structure of the voluntary contribution game.

This paper is organized as follows. Section 2 presents our experimental design and procedures, followed by predictions in Sect. 3. Section 4 discusses our experimental results, Sect. 5 concludes the paper.

\section{Experimental design and procedures}

We consider two variants of the public goods game, namely the standard linear public goods game and the threshold public goods game. In the baseline treatment we do not impose any default option. We compare this baseline treatment with the opt-out treatment, where by default subjects are deemed to agree to make non-zero contribution to the public goods provision, and the opt-in treatment, where by default subjects do not contribute to the public goods provision. We ran these three experimental treatments in both the linear and the threshold public goods game. 
Subjects formed groups of 4 and played the public goods game over 10 rounds with the first 2 rounds being the trial rounds. In every round, subjects were randomly rematched. At the beginning of each round, subjects were given 100 endowment points, which they could allocate to their own private account, or to the group account, or any combination of both.

In the linear public goods game, the payoff for each subject can be expressed as

$$
\pi_{i}=E_{i}-c_{i}+\alpha \sum_{i=1}^{4} c_{i}
$$

where, $E_{i}, c_{i}$, and $\alpha$ denote, respectively, player $i$ 's initial endowment, which is set at 100 points, player $i$ 's contribution to the group account, and the marginal per-capita return (MPCR), which is set at 0.5 . Given that the MPCR from placing 1 point in the group account is less than the marginal return from placing it in the individual private account $\left(E_{i}-c_{i}\right)$, i.e., $\partial \pi_{i} / \partial c_{i}=-1+\alpha<0$, the dominant strategy for a player would be to place all points in the private account and to let others contribute to the group account. Anticipating this behavior, other players would follow suit. As a result, in equilibrium, no one would make any contribution to the group account. Zero contribution is the unique and inefficient Nash equilibrium in this linear public goods game.

In the threshold public goods game, the public goods will be provided if and only if the group contribution threshold $(T)$ is met. If the threshold is not met, the public goods will not be provided and the points contributed in the group account will be refunded subject to the refund rate of $\mathrm{r}$ per contribution point. The payoff for each subject $\left(\pi_{i}\right)$ can then be expressed as

$$
\pi_{i}=\left\{\begin{array}{lc}
E_{i}-c_{i}+\alpha \sum_{i=1}^{4} c_{i} & \text { if } \sum_{i=1}^{4} c_{i} \geq T \\
E_{i}-c_{i}+r c_{i} & \text { if } \sum_{i=1}^{4} c_{i}<T
\end{array}\right.
$$

We set the threshold T equal to 200 points and that the refund rate is 0.5 . In the threshold public goods game, there will be multiple pure-strategy equilibria containing the inefficient zero-contribution equilibrium, and a set of efficient equilibria that includes all possible combinations of contributions where the threshold is exactly satisfied. Among these efficient equilibria, there exists a unique symmetric equilibrium where everybody contributes exactly 50 points. This symmetric equilibrium serves as a focal point that helps players coordinate their contribution decisions.

Subjects were informed of the total amount of contributions collected in the group account and their individual earnings from a particular round at the end of that round. 1 out of 8 real rounds was randomly selected as the binding round to determine their payment. The points earned were converted into Singapore Dollars at the rate of 1 point $=0.10$ SGD.

In the opt-out treatment, by default subjects were considered to have chosen to contribute to the group account. This implied that, as long as they did not decide to opt-out from the default option, they were required to make non-zero contribution 
to the group account. In other words, the default option in this setup is only related to the decision on whether or not to participate in the provision of public goods, but not on the amount of contribution. Further, the default option in the opt-out treatment also has a positive connotation from the view point of society as it encourages people to participate in public goods provision. For this reason, we label the automaticparticipation default-option in the opt-out treatment as the positive default in this paper. In contrast to Altmann and Falk (2009) and Carlsson et al. (2015), the default option in our opt-out treatment did not require subjects to contribute their entire endowment to the group account. They could, in principle, contribute an amount that was slightly above zero, which in essence was equivalent to no contribution. Our experimental design, therefore, maintains the voluntary nature of the public goods game.

In the opt-in treatment, by default subjects were considered to have chosen not to contribute at all to the group account, but they can opt in to the contribution plan. The zero-contribution default has a negative connotation from the society's perspective as it essentially uses the free-riding equilibrium as the default option. We label the no-participation default-option in the opt-in treatment as the negative default in this paper.

We employed both types of the public goods game because the effect of defaults may vary due to the different structure of the game. Studying both gives us a more comprehensive picture of the effects of defaults. In the linear public goods game, the unique pure strategy Nash equilibrium is characterized by players making zero contribution. Rational selfish models give clear predictions on the contributing behavior. In contrast, in the threshold public goods game there are multiple Nash equilibria that include the zero contribution equilibrium and the positive interior contribution equilibria. It is unclear what decision rational subjects would prefer to take in this game when no default option is used. Any of these equilibria is possible (Palfrey and Rosenthal 1984). It would be intriguing to explore if and in what way the default effect varies according to the structure of the game.

The experiment was conducted at Nanyang Technological University (NTU). The subjects were undergraduate students from various majors. The linear public goods game experiments were first conducted in February, 2011, and were programmed using a web-based interactive program. The threshold public goods game experiments were conducted in October, 2012, and were programmed using Z-tree (Fischbacher 2007). ${ }^{1}$ Another 6 new experimental sessions were run in June, 2016. In each experimental session we grouped subjects into 2 independent clusters, and across rounds subjects were only randomly re-matched with other subjects belonging to the same cluster. This way, we were able to have 2 independent observations at the group level in each session. All in all, we have 284 subjects participating in 12 experimental sessions and each treatment has 3 independent cluster level observations ( 1 from the previously done experiment and 2 from the recently done experiment). Table 1 summarizes the basic descriptive statistics of participants. The experimental instructions were read

\footnotetext{
1 It should be noted that in this paper we do not compare treatments across games. That is, for example, we do not compare the opt-out treatment in the linear public good game and the opt-out treatment in the threshold public good game.
} 
Table 1 Descriptive statistics by game and treatment

\begin{tabular}{llllll}
\hline Game type & Treatment & Subject & Observation & Mean gender (Male=1) & Mean age (SD) \\
\hline \multirow{2}{*}{ Linear } & Control & 44 & 352 & 0.48 & $21.20(1.77)$ \\
& Opt-out & 48 & 384 & 0.63 & $20.88(1.72)$ \\
& Opt-in & 48 & 384 & 0.54 & $21.54(1.4)$ \\
\multirow{4}{*}{ Threshold } & Control & 48 & 384 & 0.42 & $20.91(1.90)$ \\
& Opt-out & 48 & 384 & 0.58 & $21.33(1.91)$ \\
& Opt-in & 48 & 384 & 0.50 & $21.38(1.98)$ \\
\hline
\end{tabular}

aloud to the subjects. ${ }^{2}$ At the end of the experiment, the participants were asked to fill in a post-experiment questionnaire before being handed in their earnings from the experiment.

\section{Experimental predictions}

In this section, we formulate some experimental predictions. The predictions focus on two aspects, that is, subjects stay with the default options or make active decisions, and the effects of default options on contribution levels relative to those generated in the control treatment whereby default options are absent. We formulate the rational (standard economic theory) based prediction and the behavioral based prediction for each aspect.

Prediction 1a (Rational Selfish Prediction). Subjects faced with the positive default in the opt-out treatment will take active decisions (switch) in the linear public goods game and stay with default options in the threshold public goods game. Subjects faced with negative defaults in the opt-in treatment will always stay with default options in both games.

The unique Nash equilibrium in the linear public goods game is the zerocontribution equilibrium. Standard economic theory suggests that subjects will always make a zero-contribution regardless of the defaults. In other words, subjects tend to go against the cooperative default in the opt-out treatment and stay with the noncooperative default in the opt-in treatment in order to reach the equilibrium in the linear public goods game.

There are multiple equilibria including the inefficient zero-contribution equilibrium and the efficient interior equilibria in the threshold public goods game. The presence of the threshold transforms the game with a unique equilibrium to a coordination game with multiple equilibria. In such a game, the equilibria can be categorized as either risk dominant or payoff dominant (see Harsanyi and Selten 1988). A large body of literature has studied the equilibrium selection problem in coordination games both theoretically and experimentally (e.g., Huyck et al. 1990, 1991; Kim 1996; Rankin et al. 2000; Broseta et al. 2003; Schmidt et al. 2003). Yet, these studies do not give

\footnotetext{
2 The experimental instructions can be found in the appendix.
} 
Table 2 Summary of predictions of staying or switching regarding default options

\begin{tabular}{llll}
\hline & & Positive default (Opt-out) & Negative default (Opt-in) \\
\hline Linear & Rational & Switch & Stay \\
\multirow{3}{*}{ Threshold } & Behavioral & Stay & Stay \\
& Rational & Stay & Stay \\
& Behavioral & Stay & Stay \\
\hline
\end{tabular}

us any conclusive evidence on whether the risk dominant or the payoff dominant equilibrium is more likely to be selected. In particular, the equilibrium outcome is shown to be sensitive to the game context, the payoff structure, the group size and other factors.

In the threshold game, if subjects move away from the default (opt out), they have no other choice than contributing zero. If they stay with the default in the optout treatment, their action space is wider. They can essentially choose any positive contribution amount. Since the threshold game is essentially a coordination game with multiple equilibria, and any positive contribution amount that would satisfy the threshold is possible in equilibrium, staying with the default option may make the positive contribution equilibrium more salient for subjects. The default option provided could help subjects coordinate their actions on the positive contribution equilibrium. We thus postulate that rationality and the role of default as a coordination device would induce subjects to stay with the positive default in the threshold public goods game. Likewise, the negative default in the opt-in treatment would make the zero-contribution equilibrium more salient. We posit that people tend to stay with the default option as well.

Prediction 1b (Behavioral Based Prediction). Subjects will stay with default options in both games, regardless of the type of defaults.

The existing behavioral models and empirically findings in the literature suggest that the default is sticky and people are likely to stay with the exerted default option. For instance, the "status quo" bias or the relative unwillingness of people to move away from a status quo (see Samuelson and Zeckhauser 1988; Kahneman et al. 1991). This could be because moving away from the status quo entails some (psychological) costs or when people are loss averse they would be reluctant to move away from the default. The prevailing default option might be perceived as a suggestion of the course of action to follow (see Madrian and Shea 2001; Thaler and Sunstein 2008; Beshears et al. 2009). Thus, subjects will always stay with default options regardless of their type. Table 2 summarizes various prediction categories for Prediction 1.

Prediction 2a (Rational Selfish Prediction). Default options have no effect on contribution levels.

In a fully rational world, default options are not supposed to affect people's choice as people are assumed to be rationally selfish and always maximize the utility. Since default options do not change the payoff structure of the game or the equilibrium outcomes, individuals still choose the best action for them. That is to say, default options should not matter (see Thaler and Sunstein 2008; Cappelletti et al. 2014). 
Table 3 Summary of predictions of the effects of defaults on contribution levels

\begin{tabular}{llll}
\hline & & Positive default (Opt-out) & Negative default (Opt-in) \\
\hline Linear & Rational & No effect & No effect \\
& Behavioral & Positive & Negative \\
Threshold & Rational & No effect & No effect \\
& Behavioral & Positive & Negative \\
\hline
\end{tabular}

Though mounting empirically evidence suggests that this might be unrealistic, we present this prediction for completeness.

Prediction 2b (Behavioral Based Prediction). The positive default in the opt-out treatment increases the contribution level, and the negative default in the opt-in treatment has the opposite effect.

It has been proposed in empirical studies that the default option could be perceived as a suggestion or endorsement from the policy maker (see Madrian and Shea 2001; Thaler and Sunstein 2008; Beshears et al. 2009). There could be an "information leakage" from the default options, which might send normative signals of what to do (see McKenzie et al. 2006; Sher and McKenzie 2006). The positive default in the optout treatment might send a signal that people should make contributions to the public goods and therefore boosts contribution. People might infer from the negative default in the opt-in treatment that it might be acceptable not to contribute and therefore reduce contribution. Besides the endorsement hypothesis mentioned above, default options could have subtle influence on preferences as well. Dhingra et al. (2012) named this effect as "default pull". Dhingra et al. (2012) suggested that people have uncertainty over their preferences and default options could help people construct their preferences. The positive default in the opt-out treatment might encourage people to contribute, while the negative default might discourage people to contribute. Table 3 summarizes various prediction categories for Prediction 2.

\section{Experimental results}

We focus on two issues in this section; firstly, the incentive of subjects to switch from or to stay with the default options, and secondly, the impact of default options on contribution levels. In both of the following subsections, the presentations are arranged in this order. In the analysis, we will relate the results obtained to the predictions presented earlier.

\subsection{Descriptive statistics}

Table 4 summarizes the proportion of active decisions and default options taken. In the opt-out treatment, the overwhelming majority of subjects (85.7 and $83.3 \%$ in the linear and threshold public goods games, respectively) stick to the default option and contribute a non-zero amount to the group account. In contrast, in the opt-in treatment, 
the overwhelming majority of subjects (73.2 and $91.4 \%$ in the linear and threshold public goods games, respectively) do not stick to the default option and instead prefer to take an active decision to contribute. This result suggests that people did not always blindly follow the default option. When the default carries a negative impression (i.e. forcing them to deviate from the contribution 'norm' and 'labeling' them as noncooperators); they would prefer to move away from the default. However, when the default carries a positive impression (i.e. conforming to the contribution 'norm' and 'labeling' them as cooperators); they would prefer to stick with the default option. Thus, we find evidence of partial stickiness of the default option. The default option is neither irrelevant (Prediction 1a) nor completely sticky (Prediction 1b).

In the linear public goods game, the average contribution in the opt-out treatment was visibly higher than that in the control treatment. It was, however, substantially lower in the opt-in treatment than that in the control treatment. The results in the linear public goods game thus were not in line with the rational selfish prediction stated in Prediction 2a. They are however, consistent with the behavioral based prediction stated in Prediction 2b.

A different picture emerged in the threshold public goods game. The average contributions across all three treatments were slightly higher than the average contribution at the focal symmetric equilibrium outcome where everybody contributes 50 points to meet the threshold. The average contributions across treatments were more or less similar while the contribution in the opt-out treatment became a bit lower than the other two. The results in the threshold public goods game were partially consistent with Prediction $2 \mathrm{a}$ but not with Prediction $2 \mathrm{~b}$. In general, subjects showed a stronger motivation to contribute in the threshold public goods game regardless of the nature of the default option employed.

Besides the average contribution, we also present the conditional average contribution and the percentage of non-zero contributions across treatments (see Table 4). The presence of the default option, regardless of its nature, boosts the participation rate in the linear public goods game relative to the control treatment. The average contribution conditional on positive contributions in the opt-out treatment is similar to that in the control treatment while it is much higher than that in the opt-in treatment. The increased conditional average contribution in the opt-out treatment seems to be driven by the higher participation rate.

Figure 1 depicts the average contribution across rounds (excluding the first 2 trial rounds). Panel A shows that the average contribution in the opt-out treatment in the linear public goods game dropped in the early rounds, but subsequently remained relatively consistent across rounds. In contrast, in the other two treatments, the average contribution decayed across rounds. The decaying trend is consistent with findings in the existing literature (e.g., Andreoni 1995; Palfrey and Prisbrey 1997; Houser and Kurzban 2002). In addition to promoting contributions, the opt-out treatment in the linear game helps sustain contributions as well. The graph shown in Panel B, in contrast, shows that the average contribution in all treatments in the threshold game was relatively stable across rounds. It is consistent with the findings in Messer et al. (2008). The average contribution started slightly higher than 50 points, which was much higher than the starting average contribution in the linear public goods game. In 


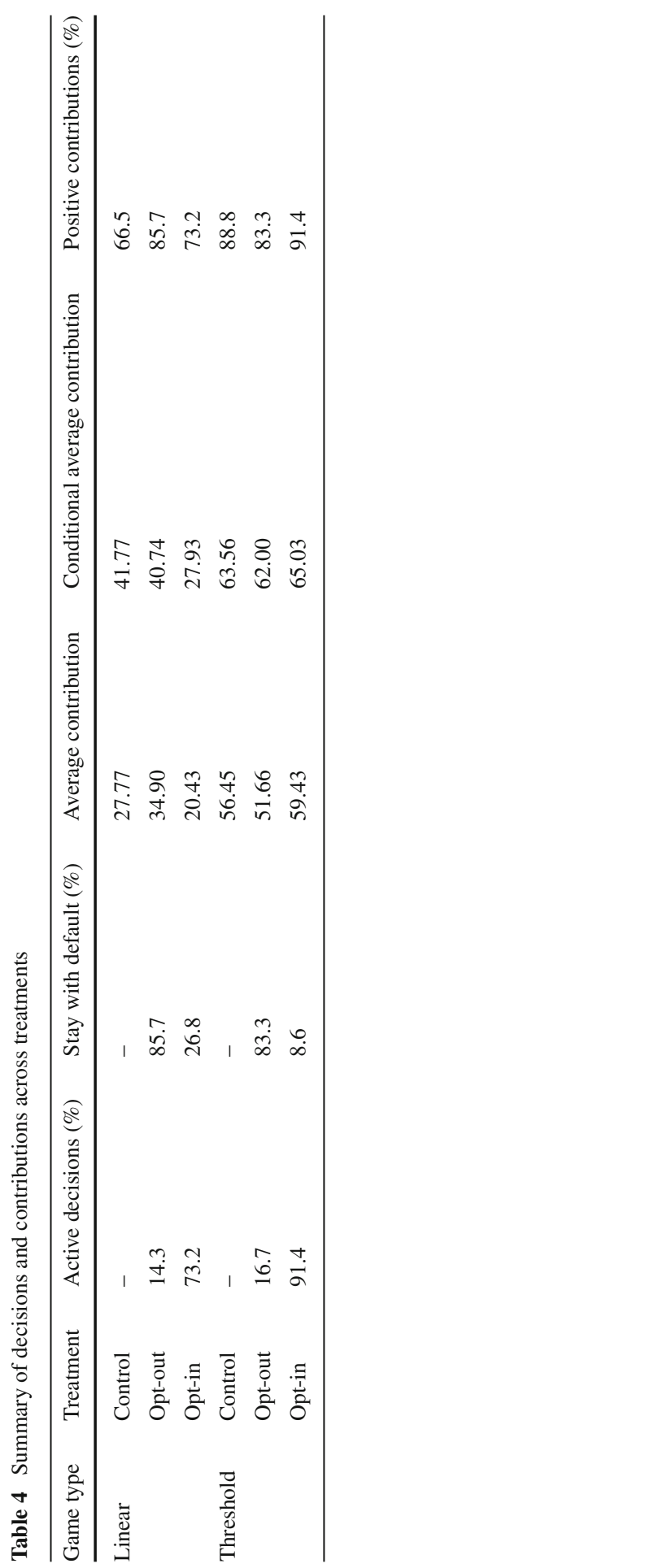


Panel A. The linear public goods game Panel B. The threshold public goods game

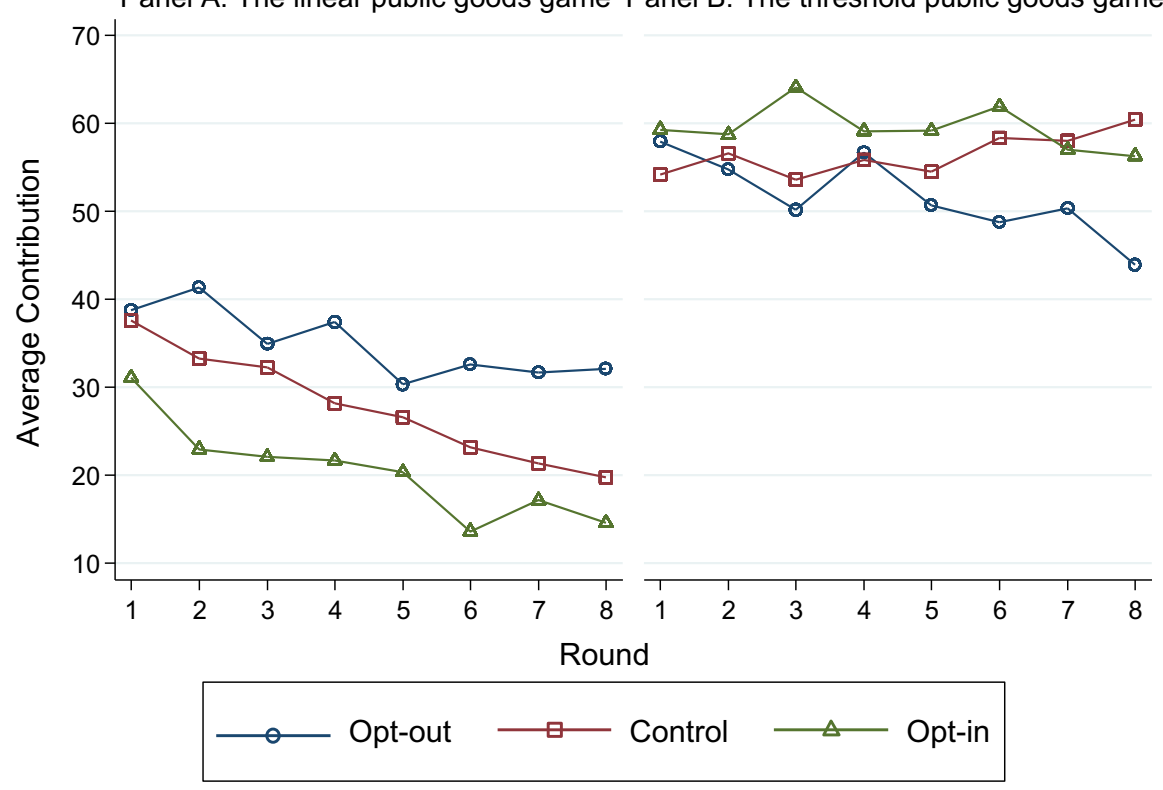

Fig. 1 Average contribution over time

contrast to the clear difference in contribution levels in the linear public goods game, the contribution level over time was less different across treatments.

Figure 2 shows the distribution of subjects' frequency of switching away from the default option in the opt-out and opt-in treatments. Panels A and B present the linear and threshold public goods games, respectively. This frequency labeled on the $\mathrm{x}$-axis is defined as the number of rounds wherein an active decision to nullify the default was taken over the total number of rounds. It can be seen that the frequency distribution of switching in the opt-out treatment was markedly different from that in the opt-in treatment, regardless of the type of the public goods game employed. This confirms our earlier result. The nature of the default option does matter for subjects' decisions on whether or not to stay with the default option.

About $65 \%$ of subjects in the opt-out treatment never switched from the default participation option in the linear public goods game. The proportion stood at around $60 \%$ in the threshold public goods game. In contrast, about $50 \%$ of subjects in the opt-in treatment always switched from the default participation option in the linear public goods game. The proportion was much higher $(73 \%)$ in the threshold public goods game. It is interesting to note that the distributions of the frequency of switching in both treatments in the threshold public goods game had very little overlapping. This further corroborates our earlier finding that people seemed to have stronger motivation to contribute to public goods in the threshold public goods game.

Figure 3 presents the distributions of subjects' average contributions in all treatments in both games. The vertical axis represents the percentage of subjects whose average contributions fall into the range of average contribution specified in the hor- 

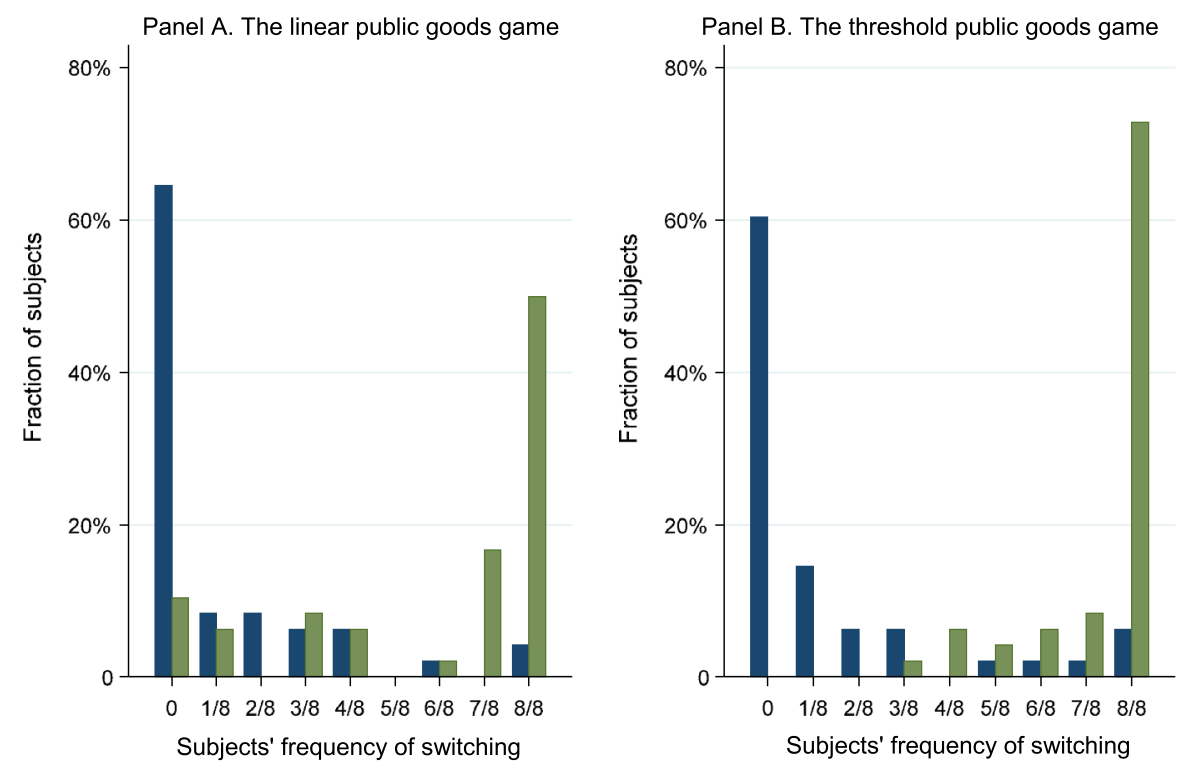

\begin{tabular}{|l|l|}
\hline Opt-out & Opt-in \\
\hline
\end{tabular}

Fig. 2 The distribution of subjects' frequency of switching
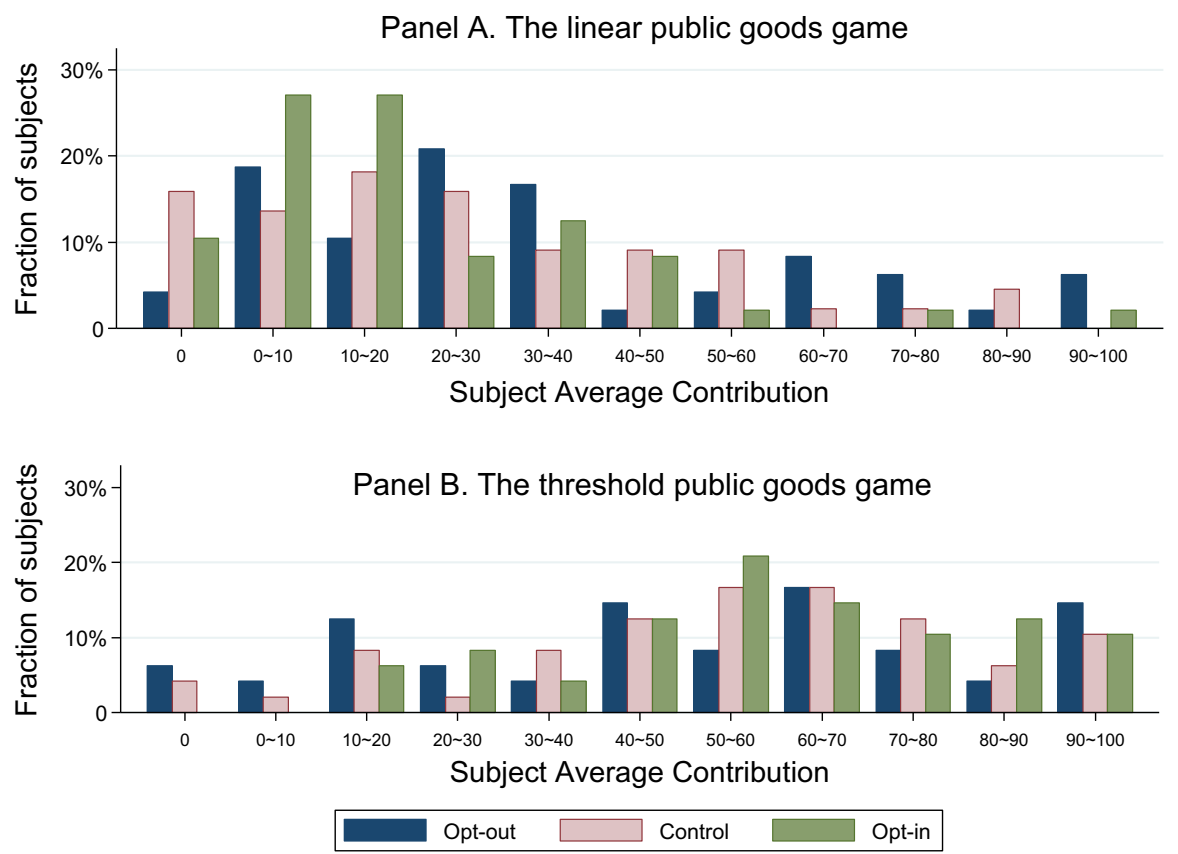

Fig. 3 The distribution of subjects' average contributions 
izontal axis. It can be seen that the distributions in the two public goods games were substantially different.

Note that in the linear public goods game shown in Panel A, the zero contribution bar in the opt-out treatment represents the proportion of subjects who decided to optout from participating in the public goods contribution in all rounds, while that in the opt-in treatment represents those who always decided to stay with the default option of no participation. It seems that the positive default in the opt-out treatment discourages zero-contributions compared to that in the control treatment. Panel A also shows that in the opt-out treatment roughly around $27 \%$ of subjects contributed more than 50 points, while in the control and opt-in treatments only around 18 and $6 \%$ of subjects did so, respectively. When we compare the opt-out and control treatments, we can see that the distribution of contributions in the opt-out treatment tended to shift to the right relative to that in the control treatment.

Panel B shows the distribution of contributions across treatments in the threshold public goods game. Subjects' average contributions are clustered around a higher contribution range in all treatments. This suggests that the presence of the threshold generally decreased selfish behaviors. The use of the default option did not seem to increase contributions in the threshold public goods game. We also found that the distributions of contributions were similar across treatments.

\subsection{Regressions}

Next, we further examine the determinants of active decision and individual contribution through regression analyses. Table 5 presents the multilevel mixed-effects probit estimation of the determinants of active decision, which takes a value of 1 if the subject goes against the default option to make a zero-contribution in the opt-out treatment or to make a positive contribution in the opt-in treatment, and takes a value of 0 otherwise. Panels A and B represent separate regression results for the linear public goods game and the threshold public goods game, respectively. The explanatory variables include the treatment dummy for the opt-out treatment (Opt-out treatment) with the opt-in treatment being the baseline treatment, contribution in the previous round (Contribution $(t-1))$, the interaction between the opt-out treatment dummy and contribution in the previous round (Opt-out * Contribution $(t-1)$ ), individuals' earnings in the previous round (Earnings $(t-1)$ ), time trend (Round), the indicator for new sessions (Wave) taking value 1 for new sessions, age (Age), and gender (Gender) taking the value of 1 for male and 0 otherwise. In addition, an indicator variable which takes a value of 1 if the threshold in the previous round was reached and 0 otherwise is included in the panel $\mathrm{B}$ regression. The lagged variables are included to capture the effect of past outcomes on the contribution decision in the current round.

The signs of the treatment dummy and the interaction term with the treatment dummy are negative indicating that, relative to the opt-in treatment, subjects in the opt-out treatment are less likely to take an active decision in both the linear public goods game and the threshold public goods game. This confirms our earlier conclusion that the nature of the default option matters. Subjects are more likely to stay with the default option when it is consistent with their inherent motivation, instead of blindly 
Table 5 Determinants of active decision: multilevel mixed-effects probit estimation

\begin{tabular}{|c|c|c|}
\hline & \multicolumn{2}{|c|}{ Dependent variable: active decision } \\
\hline & Panel A linear & Panel B threshold \\
\hline Opt-out treatment & $\begin{array}{l}-1.098^{* * *} \\
(0.163)\end{array}$ & $\begin{array}{l}-0.354 \\
(0.340)\end{array}$ \\
\hline Contribution $(t-1)$ & $\begin{array}{l}0.022 * * * \\
(0.004)\end{array}$ & $\begin{array}{l}0.033 * * * \\
(0.007)\end{array}$ \\
\hline Opt-out $\times$ Contribution $(t-1)$ & $\begin{array}{l}-0.029 * * * \\
(0.005)\end{array}$ & $\begin{array}{l}-0.047 * * * \\
(0.007)\end{array}$ \\
\hline Earnings $(t-1)$ & $\begin{array}{l}0.004 * \\
(0.002)\end{array}$ & $\begin{array}{l}0.012 * * \\
(0.005)\end{array}$ \\
\hline Round & $\begin{array}{l}0.033 \\
(0.030)\end{array}$ & $\begin{array}{l}-0.010 \\
(0.030)\end{array}$ \\
\hline Wave & $\begin{array}{l}-0.380^{* * * *} \\
(0.127)\end{array}$ & $\begin{array}{l}0.284 \\
(0.194)\end{array}$ \\
\hline Age & $\begin{array}{l}0.055 \\
(0.043)\end{array}$ & $\begin{array}{l}0.034 \\
(0.057)\end{array}$ \\
\hline Gender & $\begin{array}{l}-0.131 \\
(0.123)\end{array}$ & $\begin{array}{l}0.044 \\
(0.219)\end{array}$ \\
\hline Threshold $(t-1)$ & & $\begin{array}{l}-1.573 * * \\
(0.612)\end{array}$ \\
\hline Constant (fixed-effects) & $\begin{array}{l}-1.365 \\
(0.959)\end{array}$ & $\begin{array}{l}-1.731 \\
(1.330)\end{array}$ \\
\hline Constant (random-effects) & $\begin{array}{l}0.000 \\
(0.000)\end{array}$ & $\begin{array}{l}0.000 \\
(0.000)\end{array}$ \\
\hline Observations & 672 & 672 \\
\hline
\end{tabular}

*** Significant at the $1 \%$ level

** Significant at the $5 \%$ level

* Significant at the $10 \%$ level

following it. Individual contribution in the previous round has significant impacts on the probability of taking active decisions in the opt-in and opt-out treatments for both games. Subjects who made a higher contribution in the previous round are more likely to take active decisions in the opt-in treatment. In contrast, those who made a higher contribution in the previous round are less likely to take active decisions in the opt-out treatment. ${ }^{3}$ It further suggests that the tendency of taking active decisions depends on the nature of defaults implemented and the strength of motivation to contribute. This again indicates that Prediction $1 \mathrm{~b}$ does not find its support.

\footnotetext{
3 The linear combination test of Contribution $(t-1)$ and the interaction variable shows that the coefficient of Contribution $(t-1)$ in the opt-out treatment is negatively significant in the linear game, which indicates that the treatment effect is captured by the negative and significant treatment dummy and the effects on Contribution $(t-1)$. The test shows a negative and significant coefficient in the threshold game, which means that the treatment effect is captured by the effects on Contribution $(t-1)$.
} 
Table 6 Determinants of contribution: multilevel mixed-effects linear estimation

\begin{tabular}{lll}
\hline & Dependent variable: contribution & \\
\cline { 2 - 3 } & Panel A linear & Panel B threshold \\
\hline Opt-out treatment & $9.517^{* *}$ & $-5.558^{* *}$ \\
& $(2.155)$ & $(2.410)$ \\
Opt-in treatment & $-11.112^{* *}$ & 1.048 \\
& $(2.139)$ & $(2.422)$ \\
Earnings $(t-1)$ & $-0.311^{* * *}$ & $-0.135^{* * *}$ \\
Round & $(0.029)$ & $(0.024)$ \\
& $-2.417^{* * *}$ & -0.202 \\
Wave & $(0.432)$ & $(0.488)$ \\
Age & 8.051 & $-22.374^{* *}$ \\
Gender & $(10.523)$ & $(10.378)$ \\
Threshold $(t-1)$ & 0.754 & $2.693^{* * *}$ \\
Constant (fixed-effects) & $(0.575)$ & $(0.597)$ \\
Constant (random-effects) & $-3.946^{* *}$ & -2.208 \\
Observations & $(1.824)$ & $(2.108)$ \\
\hline
\end{tabular}

*** Significant at the $1 \%$ level

$* *$ Significant at the $5 \%$ level

* Significant at the $10 \%$ level

Table 6 presents the multilevel mixed-effects linear estimates of the determinants of individual contribution. Panels A and B present the linear and threshold public goods games, respectively. The regressors include: treatment dummies for the opt-out treatment (Opt-out treatment) and the opt-in treatment (Opt-in treatment), individuals' earnings in the previous round (Earnings $(t-1)$ ), time trend (Round), the indicator for new sessions (Wave), age (Age), gender (Gender) which takes the value of 1 for male and 0 otherwise, and a dummy variable indicating whether the threshold was met in the previous round (Threshold $(t-1))$. The control treatment serves as the baseline.

It can be seen that being in the opt-out treatment led to an increase of around 10 points in contribution relative to that being in the control treatment in the linear game (Panel A). This impact is statistically significant at the 5\% level. The regression results are consistent with findings in Fig. 1. It is important to note that if subjects preferred to stay with the default option in our opt-out treatment, they would need to make a non-zero contribution to the public goods provision. The amount of contribution that subjects had to make was not specified so as to maintain the voluntary nature of 
contributions made by subjects. This is an important aspect that distinguishes our setup from those in the studies conducted by Altmann and Falk (2009) and Carlsson et al. (2015). In other words, our default option is on the participation in the provision of public goods, rather than on the amount of contribution. The use of zero-contribution defaults has significantly negative impacts on the contribution level. The results are in line with part of Prediction $2 \mathrm{~b}$ concerning the linear public goods game.

In the threshold public goods game (Panel B), the use of default participation options did not have as strong effects on subjects' contributions as that in the linear game. Though the opt-out treatment dummy is surprisingly negatively significant. The use of zero-contribution default has no effects on contributions. As mentioned earlier, this could be due to the fact that the threshold itself serves as a powerful coordination device for subjects to make a collective contribution that would meet the threshold. This effect rendered the use of a non-binding default option redundant. The evidence does not support Prediction $2 \mathrm{~b}$ in relation to the threshold public goods game.

The negative effects on contribution in the opt-out treatment in the threshold public goods game are interesting and a bit puzzling. As there are multiple equilibria in this game, none of which coincides with the socially optimal outcome, beside facing the dilemma between equilibria and the socially optimal outcome, people also face the choice among multiple equilibria. The positive default in the opt-out treatment might increase people's belief about others' contributions. After being disappointed by not higher contributions in early rounds, people might respond by cutting contributions (Bell 1985). This might help explain why the average contribution in Fig. 1 only starts to decline in later rounds. Alternatively, the contributing default requiring people to actively decide on the contribution level might add an additional layer of complications to the context and might make people focus more on meeting the threshold instead of achieving the socially optimal outcome. It might create more confusion in terms of equilibrium selection as coordination failure is so common (see Devetag and Ortmann 2007). To the best of our knowledge, there has not been any studies exploring the use of defaults in social dilemmas involving multiple equilibria as well. Unfortunately, the current design is not able to give a clear answer in this regard. Exploring the channels through which defaults work in such a context is beyond the scope of this paper. It might be a promising area for future research.

\section{Concluding remarks}

In this paper, we tested the robustness of the evidence showing that people are reluctant to switch from a given non-binding default option and evaluated the impact of nonbinding default participation options on the incentive of people to cooperate in the provision of public goods. It is noteworthy that we only specify the nature of defaults, that is, the participation and no-participation default. Subjects still have to decide how much to contribute if they stick to the participation default. In other words, the default exerted in our experiment is a hybrid system of active decisions and standard defaults which require no additional decision if those default options are taken.

Our paper is closely related to Cappelletti et al. (2014) but is different from Cappelletti et al. (2014) in several aspects. Cappelletti et al. (2014) found that the use of 
default contribution is preferred to simple contribution advice. It should be noted that in their paper, the default contribution adopted is analogous to our opt-in treatment but not to our opt-out treatment. They showed that default option is sticky. Their paper's objective was not to explore whether other types of defaults would also be sticky. Therefore, they did not compare different types of default options. In contrast, in this paper, by varying the default options provided, we are able to infer that not all default options have the same effect. In particular, we showed that the default option in the form of opt-out was sticky, but that in the form of opt-in was not.

We also examined the extent to which these defaults affect cooperative behavior in different contexts. In particular, we focused on the voluntary contribution game in which people collectively provide public goods for the benefit of everybody in the group. In such a setting, one person's decision has a bearing on other people's well-being. We implemented a linear public goods game where a unique inefficient equilibrium existed and a threshold public goods game in which there were an inefficient zero-contribution equilibrium and a set of efficient equilibria. The most efficient outcome for the group as a whole involves all group members making a full contribution; unfortunately, the socially optimal outcome is hard to achieve.

The evidence presented in the preceding sections indicates that people do respond differently according to the type of default in both games instead of blindly following it. However, the effects of non-binding defaults on contribution, to a large degree, depend on the context. Whether people take active decisions or not depends on their motivation to contribute. The frequency of staying with the participation default and the frequency of taking active decisions against the no participation default are both higher when subjects have stronger motivation to contribute. On one hand, non-binding defaults could be influential in affecting the contribution amount; on the other, there are certain limitations. The nature of default options matters in terms of affecting contributions in the linear public goods game, even when the games played are essentially the same. Our experimental results show that the cooperative default substantially increases contributions in the linear public goods game. In addition, both types of default options increase the participation rate to some degree in the linear game. However, we did not find similar effects of defaults on individual contribution in the threshold public goods game, which sheds light on the limitation of defaults. The threshold itself acts as a powerful coordination device and its presence might interact with non-binding defaults to create surprising sometimes undesirable effects.

Our findings contribute to the literature on the use of defaults in public goods game, among which our paper especially relates to a recent paper Carlsson et al. (2015). Carlsson et al. (2015) found that the zero-contribution default significantly decreased contribution, and the full-contribution default did not have much effect on contribution. Several factors could explain the difference between their results and ours. Firstly, there is a difference in the structure of the threshold game. Carlsson et al. (2015) employed a threshold public goods game with a full refund policy and proportional rebate rule and people played the game in large groups. In our study, we employed a partial refund policy and no rebate rule, and the group size was substantially smaller. As suggested by Croson and Marks (2000), these three factors: the refund policy, the rebate rule and the group size, influence the extent of which default settings affect the contribution amount. Secondly, the default option employed in the opt-out treatment in their study 
was different from ours. The default option employed in Carlsson et al. (2015) was the default contribution amount, while the default option employed in our study was the default on the participation decision. Participants in our study still needed to decide their contribution amount should they decide to participate in the provision of public goods. Thirdly, in Carlsson et al. (2015) the experiment was conducted in a Vietnamese village and at the subjects' place of residence. As such, the condition of anonymity in their study was weakened. Subjects could feel that they were being observed by the researchers when making their decisions, and this may, to some degree, influence their decisions.

Acknowledgements This research benefited from the start-up Grant (SUG) and Tier 1 Grant awarded to Yohanes E. Riyanto by Nanyang Technological University.

\section{Compliance with ethical standards}

Conflicts of interest The authors declare that they have no conflict of interest.

Open Access This article is distributed under the terms of the Creative Commons Attribution 4.0 International License (http://creativecommons.org/licenses/by/4.0/), which permits unrestricted use, distribution, and reproduction in any medium, provided you give appropriate credit to the original author(s) and the source, provide a link to the Creative Commons license, and indicate if changes were made.

\section{References}

Altmann S, Falk A (2009) The impact of cooperation defaults on voluntary contributions to public goods. Working paper, University of Bonn

Andreoni J (1995) Cooperation in public-goods experiments: kindness or confusion? Am Econ Rev 85(4):891-904

Bagnoli M, Lipman BL (1989) Provision of public goods: fully implementing the core through private contributions. Rev Econ Stud 56(4):583-601

Bell DE (1985) Disappointment in decision making under uncertainty. Oper Res 33(1):1-27

Beshears J, Choi JJ, Laibson D, Madrian BC (2009) The importance of default options for retirement saving outcomes: evidence from the united states. Social security policy in a changing environment. University of Chicago Press, Chicago, pp 167-195

Broseta B, Fatas E, Neugebauer T (2003) Asset markets and equilibrium selection in public goods games with provision points: an experimental study. Econ Inq 41(4):574-591

Cappelletti D, Mittone L, Ploner M (2014) Are default contributions sticky? An experimental analysis of defaults in public goods provision. J Econ Behav Organ 108:331-342

Carlsson F, Johansson-Stenman O, Nam PK (2015) Funding a new bridge in rural Vietnam: a field experiment on social influence and default contributions. Oxf Econ Pap 67(4):987-1014

Carroll GD, Choi JJ, Laibson D, Madrian BC, Metrick A (2009) Optimal defaults and active decisions. Q J Econ 124(4):1639-1674

Choi J, Laibson D, Madrian B (2004) Plan design and 401 (k) savings outcomes. Technical report, National Bureau of Economic Research

Croson RT, Marks MB (2000) Step returns in threshold public goods: a meta-and experimental analysis. Exp Econ 2(3):239-259

Devetag G, Ortmann A (2007) When and why? A critical survey on coordination failure in the laboratory. Exp Econ 10(3):331-344

Dhingra N, Gorn Z, Kener A, Dana J (2012) The default pull: an experimental demonstration of subtle default effects on preferences. Judgm Decis Mak 7(1):69

Fischbacher U (2007) z-Tree: Zurich toolbox for ready-made economic experiments. Exp Econ 10(2):171178 
Harsanyi JC, Selten R (1988) A general theory of equilibrium selection in games, vol 1. The MIT Press, Cambridge

Houser D, Kurzban R (2002) Revisiting kindness and confusion in public goods experiments. Am Econ Rev 92(4):1062-1069

Johnson E, Hershey J, Meszaros J, Kunreuther H (1993) Framing, probability distortions, and insurance decisions. J Risk Uncertain 7(1):35-51

Johnson E, Goldstein D (2003) Do defaults save lives? Science 302(5649):1338-1339

Kahneman D, Knetsch J, Thaler R (1991) Anomalies: the endowment effect, loss aversion, and status quo bias. J Econ Perspect 5(1):193-206

Kim Y (1996) Equilibrium selection inn-person coordination games. Games Econ Behav 15(2):203-227

Madrian B, Shea D (2001) The power of suggestion: inertia in 401 (k) participation and savings behavior. Q J Econ 117(1):377

McKenzie CR, Liersch MJ, Finkelstein SR (2006) Recommendations implicit in policy defaults. Psychol Sci 17(5):414-420

Messer KD, Zarghamee H, Kaiser HM, Schulze WD (2007) New hope for the voluntary contributions mechanism: the effects of context. J Public Econ 91(9):1783-1799

Messer KD, Kaiser HM, Schulze WD (2008) The problem of free riding in voluntary generic advertising: parallelism and possible solutions from the lab. Am J Agric Econ 90(2):540-552

Palfrey TR, Prisbrey JE (1997) Anomalous behavior in public goods experiments: How much and why? Am Econ Rev 87(5):829-846

Palfrey TR, Rosenthal H (1984) Participation and the provision of discrete public goods: a strategic analysis. J Public Econ 24(2):171-193

Rankin FW, Van Huyck JB, Battalio RC (2000) Strategic similarity and emergent conventions: evidence from similar stag hunt games. Games Econ Behav 32(2):315-337

Samuelson W, Zeckhauser R (1988) Status quo bias in decision making. J Risk Uncertain 1(1):7-59

Schmidt D, Shupp R, Walker JM, Ostrom E (2003) Playing safe in coordination games: the roles of risk dominance, payoff dominance, and history of play. Games Econ Behav 42(2):281-299

Sher S, McKenzie CR (2006) Information leakage from logically equivalent frames. Cognition 101(3):467494

Thaler R, Sunstein C (2003) Libertarian paternalism. Am Econ Rev 93(2):175-179

Thaler R, Sunstein C (2008) Nudge: improving decisions about health, wealth, and happiness. Yale University Press, New Haven

Van Huyck JB, Battalio RC, Beil RO (1990) Tacit coordination games, strategic uncertainty, and coordination failure. Am Econ Rev 80(1):234-248

Van Huyck JB, Battalio RC, Beil RO (1991) Strategic uncertainty, equilibrium selection, and coordination failure in average opinion games. Q J Econ 106(3):885-910 